\title{
NIH's Shared Instrumentation Program: The More I Know, the Better My Chances
}

\section{Klosek $\mathbf{M}^{1}$}

1. Division of Construction and Instruments, Office of Research Infrastructure Programs (ORIP), Division of Program Coordination, Planning and Strategic Initiatives, Office of the Director, National Institutes of Health, Bethesda USA.

The National Institutes of Health (NIH)'s shared instrumentation program, located within the Office of Research Infrastructure Programs (ORIP), is unique at the NIH in supporting acquisitions of the state-of-the-art commercially available instruments. Any instrumentation request must be justified by the need of NIH-funded projects, by demonstrating how access to novel technologies will enable and advance research. The use of any instrument on a shared basis is cost-effective as it benefits work of many investigators; it also facilitates access to appropriate technical expertise and support. Over time these fundamentals of the program were kept in place unchanged, while some program elements were modified to better meet demands for access to novel technologies and the needs of emerging scientific fields. This talk will describe the program's support for different technologies, including optical instruments, and outline the current program requirements and guidelines.

"In the last five years, ORIP has awarded over 500 S10 instruments. The S10 instruments are awarded to research institutions throughout the nation to meet the needs of NIH-funded investigators and other biomedical researchers, as reflected by the equal proportion of instruments and NIH-funded research projects in each of the nation's four geographical regions" (Figure 1). (https://orip.nih.gov/construction-and-instruments/s10-instrumentation-programs) "The S10 program supports state-of-the-art technologies needed by NIH-funded investigators, by funding instruments across a wide range of categories in response to requests (Figure 2)."

\section{References:}

[1] https://orip.nih.gov/construction-and-instruments/s10-instrumentation-programs 

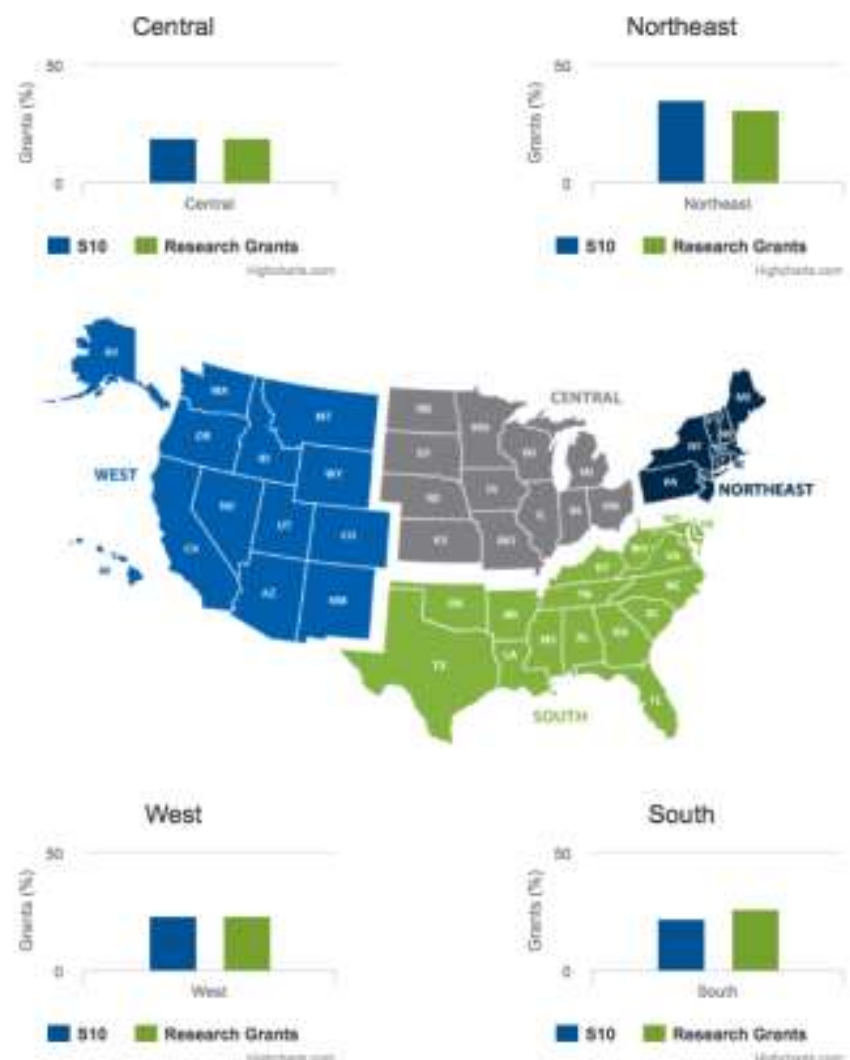

Figure 1. Map of United States divided into regions indicating the proportion of instruments to $\mathrm{NIH}$-funded research projects. https://orip.nih.gov/construction-and-instruments/s10instrumentation-programs

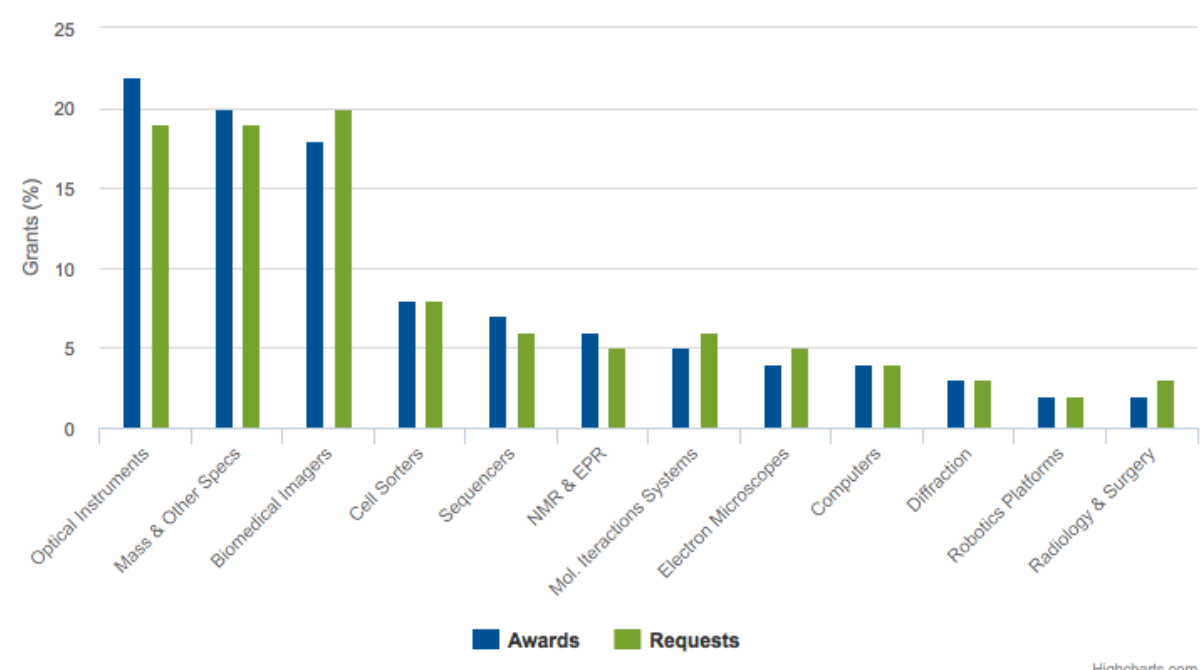

Figure 2. Percentage of each type of instrument category awarded.

https://orip.nih.gov/construction-and-instruments/s10-instrumentation-programs 